\title{
The endonuclease activity of RNase III is required for the regulation of antibiotic production by Streptomyces coelicolor
}

Correspondence

George H. Jones

george.h.jones@emory.edu

Received 9 July 2008

Revised 11 August 2008

Accepted 20 August 2008

\author{
Marcha L. Gravenbeek and George H. Jones \\ Department of Biology, Emory University, Atlanta, GA 30319, USA
}

\begin{abstract}
The double strand-specific endoRNase RNase III globally regulates the production of antibiotics by Streptomyces coelicolor. We have undertaken studies to determine whether the endoRNase activity of S. coelicolor RNase III or its RNA binding activity is responsible for its regulatory function. We show that an rnc null mutant of S. coelicolor M145 does not produce actinorhodin or undecylprodigiosin. Restoring a wild-type copy of $r n c$ to that mutant also restored antibiotic production. We constructed an rnc point mutant, D70A, in which an aspartic acid residue which is essential for the catalytic activity of RNase III was changed to alanine. The D70A mutation abolished the catalytic activity of the protein but not its ability to bind to RNA substrates. Introduction of a copy of the D70A gene into the rnc null mutant did not restore antibiotic production. This result suggests that the endoRNase activity of RNase III is required for the regulation of antibiotic production in S. coelicolor. We also reconstructed the $\mathrm{C} 120$ point mutation that was originally described in 1992. Although that mutation diminished antibiotic production by S. coelicolor, we confirm here that the C120 protein retains some RNase III activity.
\end{abstract}

\section{INTRODUCTION}

RNase III is a double strand-specific endonuclease that is found in bacteria and eukaryotes (Drider \& Condon, 2004; Ji, 2006; Nicholson, 1999). In addition to its general role in RNA processing, RNase III is involved in the regulation of gene expression in Escherichia coli and other bacteria. Thus, in E. coli RNase III autoregulates its own expression by cleaving an untranslated leader in its mRNA. This cleavage leads to an increased rate of decay of the RNase III mRNA, downregulating expression of the RNase III gene $r n c$ (Bardwell et al., 1989; Matsunaga et al., 1996, 1997). RNase III is also involved in regulation of the expression of the polynucleotide phosphorylase (PNPase) gene $p n p$ in bacteria. RNase III cleaves a stem-loop structure in the $5^{\prime}$ leader of the pnp transcripts in E. coli. The $3^{\prime}$ ends produced by this cleavage then serve as targets for polyadenylation by $\operatorname{poly}(\mathrm{A})$ polymerase I, and the polyadenylated $3^{\prime}$ ends are degraded by PNPase itself (Jarrige et al., 2001; Robert-Le Meur \& Portier, 1994). Thus, RNase III cleavage, polyadenylation and PNPase action on its own mRNA lead to instability of that mRNA, resulting in decreased synthesis of PNPase (Jarrige et al., 2001; RobertLe Meur \& Portier, 1992, 1994). There are a number of other examples of the regulation of gene expression by RNase III in E. coli and other bacteria (reviewed by Drider \& Condon, 2004; Ji, 2006; Nicholson, 1999).
RNase III also plays an important role in the regulation of antibiotic synthesis in Streptomyces coelicolor. Some years ago, Champness and co-workers identified an S. coelicolor locus, which they designated absB (Adamidis \& Champness, 1992). The synthesis of all four antibiotics normally produced by $S$. coelicolor is severely reduced in an $a b s B$ mutant, C120. Specifically, they reported that the production of actinorhodin (act) by the C120 mutant is reduced to only $2 \%$ of the normally observed level and that the level of undecylprodigiosin (red) is only $15 \%$ of that normally observed (Adamidis \& Champness, 1992). Production of the calcium-dependent antibiotic and of methylenomycin is also reduced. The same group subsequently demonstrated that the $a b s B$ locus encodes a homologue of RNase III, the double strand-specific endoRNase discussed above, and the C120 absB mutant was shown to contain a point mutation that results in a change of leucine to proline in the RNase III amino acid sequence (Price et al., 1999). They demonstrated further that antibiotic levels are even lower in an RNase III disruptant than in C120 (Price et al., 1999), a result that was recently confirmed (Sello \& Buttner, 2008). Moreover, Aceti \& Champness (1998) demonstrated decreased levels of the actII-orf4 and redD transcripts, which encode the pathway-specific regulators or SARPs (Streptomyces antibiotic regulatory proteins) for act and red biosynthesis, respectively, in the $a b s B$ mutant. They showed further that $a b s B$ mutant strains are deficient in the processing of rRNA precursors compared with the wild-type strain (Price et al., 
1999). These data strongly suggest that the $a b s B$ locus does encode an RNase, and that in some fashion that locus functions as a global regulator of antibiotic production in $S$. coelicolor. These observations marked the first evidence for the regulation of antibiotic production at the level of RNA stability.

We have shown recently that the $a b s B$ gene identified by Champness and co-workers does indeed encode a double strand-specific endoRNase, i.e. an RNase III (Chang et al., 2005). There are two general classes of mechanisms by which RNase III might regulate antibiotic production in $S$. coelicolor. In the first, the endoRNase activity of RNase III would be required. There is some evidence, however, that RNase III can regulate gene expression in other systems by binding to dsRNA without cleaving it (reviewed by Drider \& Condon, 2004; Ji, 2006; Nicholson, 1999) (and see further below). Thus, a second class of mechanisms for the regulation of antibiotic synthesis would involve binding of RNase III to appropriate RNA targets in ways that affect the expression of genes that are critical for antibiotic production, but without cleaving those RNAs.

To distinguish between these possibilities, it seemed prudent to determine whether the endoRNase activity of RNase III is actually required for its regulation of antibiotic production in S. coelicolor. We show below that the activity is required for the regulation of act and red production. We show further that the RNase III protein bearing the C120 mutation retains some enzymic activity and cleaves a model RNA transcript identically to the wild-type protein.

\section{METHODS}

Bacterial strains, plasmids and growth media. E. coli strains DH5 $\alpha$ and BL21(DE3)pLysS were used as hosts for plasmid manipulation and protein expression, respectively. E. coli strains BW25113 and ET12567 (dam, dcm, hsdS; Flett et al., 1997) were used for gene disruption and conjugation purposes. E. coli strains were grown on Difco Nutrient Agar or Luria-Bertani medium supplemented with carbenicillin $\left(100 \mu \mathrm{g} \mathrm{ml}^{-1}\right)$ and/or kanamycin $(50 \mu \mathrm{g}$ $\mathrm{ml}^{-1}$ ), as necessary. S. coelicolor M145 (parental strain), JSE1880, JSE1851 and JSE1855 were grown on soya flour-mannitol (SFM) agar or on R2YE agar or in liquid media (Kieser et al., 2000) with viomycin $\left(30 \mu \mathrm{g} \mathrm{ml}^{-1}\right)$, nalidixic acid $\left(25 \mu \mathrm{g} \mathrm{ml}^{-1}\right)$ or apramycin $\left(50 \mu \mathrm{g} \mathrm{ml}^{-1}\right)$, as necessary. For the experiments shown in Fig. 4, strains were grown on R2YE agar containing nystatin $\left(315 \mathrm{U} \mathrm{ml}^{-1}\right)$ and appropriate antibiotics.

Construction of the rnc disruptant JSE1880. The $r n c(a b s B)$ gene was replaced by a viomycin-resistance cassette using the $\lambda$ redmediated recombination system for Streptomyces (Gust et al., 2003). Cosmid SC7A1, which bears the rnc (absB) gene SCO5572, was introduced by electroporation into E. coli strain BW25113 containing the $\lambda$ red plasmid. The viomycin-resistance cassette was excised from pIJ780 (Gust et al., 2003; Kieser et al., 2000) with EcoRI and HindIII. The cassette was used as a template for PCR and amplified using primers AbsB.red.fwd2 and AbsB.red.rev2 (Table 1). The strain carrying both the $S$. coelicolor cosmid and the $\lambda$ red plasmid was electro-transformed with the extended viomycin-resistance cassette and transformants were plated on Difco Nutrient Agar containing carbenicillin $\left(100 \mu \mathrm{g} \mathrm{ml}^{-1}\right)$, kanamycin $\left(50 \mu \mathrm{g} \mathrm{ml}^{-1}\right)$ and viomycin $\left(30 \mu \mathrm{g} \mathrm{ml}^{-1}\right)$. Larger colonies were restreaked on plates with selective antibiotics (carbenicillin, kanamycin, viomycin) to enrich the population with cells bearing the mutant cosmid. Colonies were screened by PCR with primers ABSB OUT1 and ABSB OUT2 (Table 1). The $5^{\prime}$ ends of these primers are situated approximately $100 \mathrm{bp}$ upstream and downstream of the deleted region of $r n c$. These primers produced an $1124 \mathrm{bp}$ fragment with the wild-type cosmid as template and an $1800 \mathrm{bp}$ fragment with the cosmid bearing the disrupted $r n c$ gene. DNA was prepared from a positive transformant and introduced into the methylation-deficient E. coli host ET12567 (Flett et al., 1997) containing the non-transmissible plasmid pUZ8002 (Flett et al., 1997; Gust et al., 2003; Kieser et al., 2000). After conjugation using spores of $S$. coelicolor M145, desired exconjugants $\left(r n c^{-}, v^{r}{ }^{r} \operatorname{kan}^{s}\right)$ were selected by restreaking single colonies on R2YE plates with and without kanamycin, and the identity of positive exconjugants was verified by PCR using primers ABSB OUT1 and ABSB OUT2 (Table 1). The strain bearing the disrupted $r n c$ gene was designated JSE1880.

Table 1. Primers used in this study

The bases underlined for the AbsB.red.fwd2 and AbsB.red.rev2 primers correspond to sequences in the viomycin cassette used for $\lambda$ red-mediated recombination. The remaining bases flank the $r n c(a b s)$ gene. The underlined sequences represent the NdeI sites in primers SCABSBF1 and SCABSBF2, and the BamHI sites in primers SCABSBR1 and C120BH1Rev.

\begin{tabular}{|ll|}
\hline Primer & \multicolumn{1}{c|}{ Sequence } \\
\hline AbsB.red.fwd2 & 5'-AGGTCCTCGAGGTCTGAGCGGCTGGTGAGAGGCACTGTGTGTAGG CTGGAGCTGCTTC-3' \\
AbsB.red.rev2 & 5'-GATGAGCGACGGTCCGAACGCCCGCCCCTTTGCCGGGGCATTCCG GGGATCCGTCGACC-3' \\
ABSB OUT1 & 5'-AAGCGGAAGATGTCGCGCAGCAA-3' \\
ABSB OUT2 & 5'-TCGGGCAACTCGGGCATGGCTT-3' \\
MGabsBF1 & 5'-TCGGCTATCAGCTCGAGTCCGCC-3' $^{\prime}$ \\
MGabsBR1 & 5'-GTGCCGGTGCCGTACGAGACG-3' \\
SCD70AF1 & 5'-TTCCTCGGGGCCTCCGTG-3' \\
SCABSBF1 & 5'-CTGGTGAGAGGCCATATGTCAGTC-3' \\
SCABSBF2 & 5'-AGGTCTGAGCGGCATATGAGAGCC-3' \\
SCABSBR1 & 5'-GACGGGTCAGGCGGATCCGGACGC-3' \\
SC120LPF1 & 5'-CTCCAGGAACCCACCGCAACC-3' \\
C120BH1Rev & 5'-GTTCGGATCCTGCGGTGGACGGGTCA-3' \\
\hline
\end{tabular}


Construction of expression plasmids bearing wild-type and mutant rnc genes. To construct a plasmid for expression of the wild-type $r n c$ gene, the $819 \mathrm{bp} r n c(a b s B)$ ORF was released from pJSE1801 (Chang et al., 2005) with BamHI and NdeI. The resulting fragment was cloned into pIJ8600 (Kieser et al., 2000; Sun et al., 1999) digested with the same two enzymes, producing plasmid pJSE1851. This plasmid was transferred to E. coli ET12567/pUZ8002 by electrotransformation and conjugated into $S$. coelicolor JSE1880. Integration of pJSE1851 into the JSE1880 chromosome was confirmed by selecting for viomycin- and apramycin-resistant exconjugants, and via PCR using primers ABSB OUT1 and ABSB OUT2 (Table 1). To construct the plasmid that contained a point mutation in the $r n c$ $(a b s B)$ gene, a $627 \mathrm{bp}$ fragment of the $a b s B$ region was amplified in an initial PCR. This was done with a $5^{\prime}$ primer (SCD70AF1, Table 1) that introduced an A to $\mathrm{C}$ base change in the second position of the codon for amino acid 70 in the RNase III amino acid sequence. This alteration changed the aspartic acid residue at position 70 to an alanine residue, creating the $\mathrm{D} 70 \mathrm{~A}$ mutation. The $3^{\prime}$ primer used in these experiments contained a BamHI site (SCABSBR1, Table 1). To amplify the full-length $r n c$ product, the fragment obtained from the initial PCR was gel-purified and used as a $3^{\prime}$ primer in a second PCR, together with a $5^{\prime}$ primer that introduced an NdeI site into the product (SCABSBF2, Table 1). Sequencing of the resulting D70A gene showed that it contained an additional single base change but that change did not affect the amino acid sequence of the resulting protein. The PCR fragment was cloned into pCR2.1-TOPO (Invitrogen) following the manufacturer's protocol and the construct was designated pJSE1853. The $r n c$ fragment was released from pJSE1853 by digestion with BamHI and NdeI and was cloned into pET19B (Novagen), digested with the same enzymes, to yield pJSE1854. The mutant gene was also cloned into pIJ8600 to yield pJSE1855. E. coli BL21(DE3)pLysS was transformed with pJSE1854 for overexpression of the D70A protein and ET12567/pUZ8002 was transformed with pJSE1855 for conjugation into JSE1880, as described above. PCR analysis with appropriate primers of chromosomal DNA isolated from the parent organism S. coelicolor M145, from JSE1880, and from JSE1880 containing pIJ8600, pJSE1851 and pJSE1855, verified the identity of each of the relevant strains (data not shown).

Construction of the $\mathbf{C 1 2 0}$ mutant. For the reconstruction of C120, the mutant originally isolated by Adamidis \& Champness (1992), which contained a single leucine to proline change at position 188 in the RNase III amino acid sequence, we again performed two rounds of PCR. The first round involved the amplification of a $300 \mathrm{bp}$ fragment of the $a b s B$ gene with a forward primer (SC120LPF1, Table 1) that introduced a single $T$ to $C$ change in the nucleotide sequence, and a reverse primer (C120BH1Rev) that introduced a BamHI restriction site. The fragment obtained from the first PCR was then used as a $3^{\prime}$ primer in the second round of PCR together with a $5^{\prime}$ primer (SCABSBF1, Table 1) that contained an NdeI site. The resulting $r n c$ fragment was cloned into pCR2.1-TOPO (Invitrogen), yielding plasmid pJSE1950. After sequencing to confirm the identity of the cloned insert, the mutated $r n c$ fragment was removed from pJSE1950 with BamHI and NdeI and ligated to pET19B which had also been digested with those enzymes. The resulting vector, pJSE1952, was used to transform chemically competent BL21(DE3)pLysS, which was then used to overexpress a hexahistidine-tagged version of the C120 protein. pJSE1811 (Chang et al., 2005) was utilized for the production of wild-type RNase III. Overexpressing strains were grown and proteins (wild-type RNase III, and the D70A and C120 mutant proteins) were purified as described previously (Chang et al., 2005).

RNA cleavage and electrophoretic mobility shift assays. The plasmid pJSE5600 (Chang et al., 2008), which contains the cloned rpsO-pnp intergenic region from S. coelicolor, was linearized with SpeI.
The linearized plasmid was used as the DNA template for transcription with T7 RNA polymerase. Transcription reactions were performed as previously described (Chang et al., 2005), with $\left[{ }^{32} \mathrm{P}\right] \mathrm{CTP}$ as the labelled precursor, except that no EDTA was added to reaction mixtures.

RNA cleavage assays, using the internally labelled $r p s O-p n p$ transcript as substrate, were performed as described previously (Chang et al., 2005). Immediately before the assay, RNA was heated at $100{ }^{\circ} \mathrm{C}$ for $30 \mathrm{~s}$ then placed on ice. Reaction mixtures for RNA cleavage contained $2 \mu \mathrm{g}$ transcript ( 10000 c.p.m.) and 0-750 ng enzyme, either wild-type RNase III, or the D70A and C120 mutant proteins. Cleavage products were fractionated on $7 \mathrm{M}$ urea/5\% polyacrylamide gels and were visualized by autoradiography. Kinetic assays of wildtype RNase III and the C120 mutant protein were performed as described previously (Chang et al., 2005).

Electrophoretic mobility shift assays were performed in $20 \mu \mathrm{l}$ reaction mixtures, incubated at $37{ }^{\circ} \mathrm{C}$ for $10 \mathrm{~min}$. Mixtures contained $160 \mathrm{mM} \mathrm{NaCl}, 30 \mathrm{mM}$ Tris- $\mathrm{HCl}(\mathrm{pH} 8.0), 10 \mathrm{mM} \mathrm{CaCl}_{2}, 0.1 \mathrm{mM}$

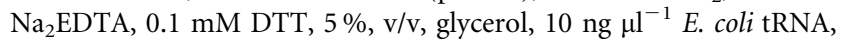
100 ng RNA (32 nM, $\sim 120000$ c.p.m., pre-heated at $100{ }^{\circ} \mathrm{C}$ for $30 \mathrm{~s}$, then placed on ice), and $20-100 \mathrm{ng}(30-160 \mathrm{nM})$ enzyme. Following incubation, the samples were immediately placed on ice for $20 \mathrm{~min}$. The $6 \%$ polyacrylamide gel was run at $75 \mathrm{~V}$ for $20 \mathrm{~min}$ and $4{ }^{\circ} \mathrm{C}$ in $0.5 \times$ Tris-borate-EDTA buffer supplemented with $10 \mathrm{mM} \mathrm{CaCl}_{2}$ prior to loading samples. Samples were then loaded on the gel and run at $85 \mathrm{~V}$ for $\sim 1.5 \mathrm{~h}$. Gels were dried and the results were visualized by autoradiography.

RT-PCR. To examine expression of the wild-type and D70A mutant $r n c$ genes in the relevant strains, RT-PCR was performed. RNA was isolated and reactions were carried out using $10 \mu \mathrm{g}$ total RNA, as described previously (Bralley \& Jones, 2003). Random hexamers (Invitrogen) were used for the reverse transcription. The cDNA was amplified using primers MGabsBF1 and MGabsBR1 (Table 1), which are specific for $S$. coelicolor $r n c$, and products were displayed on agarose gels.

Miscellaneous methods. Protein was quantified with the Bio-Rad Protein Assay, using BSA as a standard. SDS-PAGE was performed on $12.5 \%$ gels as described by Laemmli (1970), and gels were stained with Coomassie brilliant blue. PCR was performed using the Expand High Fidelity PCR System (Roche). Amplifications were generally done at an annealing temperature of $58{ }^{\circ} \mathrm{C}$. A list of plasmids used or constructed in this study is presented in Table 2.

\section{RESULTS}

\section{Construction and properties of JSE1880, pJSE1851/JSE1880 and pJSE1855/JSE1880}

As indicated above, the C120 mutant isolated by Champness and co-workers contained a point mutation in the RNase III gene rnc (Price et al., 1999). Those workers also constructed a disruptant by mutational cloning which contained two partial copies of $r n c$. For our studies, we felt it necessary to construct a true $a b s B$ null mutant. To that end, the RNase III gene was replaced with a viomycinresistance cassette in cosmid SC7A1 via PCR-targeted mutagenesis (Gust et al., 2003). The cosmid containing the disrupted gene was transferred to S. coelicolor M145 by conjugation from E. coli. Several exconjugants were examined by PCR and one such strain, containing a 
Table 2. Plasmids used or constructed in this study

\begin{tabular}{|c|c|c|}
\hline Plasmid & Properties & Source or reference \\
\hline pIJ780 & Template plasmid; $\mathrm{amp}^{\mathrm{r}}$, vio ${ }^{\mathrm{r}}$ & Gust et al. (2003) \\
\hline pUZ8002 & Non-transmissible oriT mobilizing plasmid; $\operatorname{kan}^{\mathrm{r}}$ & Kieser et al. (2000) \\
\hline pIJ8600 & Streptomycete integrative expression vector; thio $^{r}$, apra $^{r}$ & $\begin{array}{l}\text { Kieser et al. (2000); Sun et al. } \\
\text { (1999) }\end{array}$ \\
\hline pJSE1801 & S. coelicolor rnc cloned into pET11A (Novagen) & Chang et al. (2005) \\
\hline pJSE1811 & S. coelicolor rnc cloned into pET19B (Novagen) & Chang et al. (2005) \\
\hline pJSE1851 & S. coelicolor rnc cloned into pIJ8600 & This study \\
\hline pJSE1853 & S. coelicolor rnc D70A cloned into pCR2.1 TOPO (Invitrogen) & This study \\
\hline pJSE1854 & S. coelicolor rnc D70A cloned into pET19B (Novagen) & This study \\
\hline pJSE1855 & S. coelicolor rnc D70A cloned into pIJ8600 & This study \\
\hline pJSE1950 & S. coelicolor rnc $\mathrm{C} 120$ cloned into pCR2.1 TOPO (Invitrogen) & This study \\
\hline pJSE1952 & S. coelicolor rnc C120 cloned into pET19B (Novagen) & This study \\
\hline pJSE5600 & S. coelicolor 459 bp rpsO-pnp intergenic region cloned into pCR2.1 TOPO (Invitrogen) & Chang et al. (2005) \\
\hline
\end{tabular}

disrupted chromosomal copy of $r n c$, was designated JSE1880.

A copy of wild-type $r n c$ was prepared via PCR and cloned into the streptomycete expression vector pIJ8600, as described in Methods. This vector contains the thiostrepton-inducible tipA promoter (Kieser et al., 2000; Sun et al., 1999), and the plasmid resulting from the insertion of $r n c$, designated pJSE1851, was transferred to JSE1880 by conjugation from $E$. coli.

To determine whether the endoRNase activity of $S$. coelicolor RNase III was required for the regulation of antibiotic production, it was necessary to construct a mutant gene whose product lacked that activity but retained other relevant biological functions. Nicholson and co-workers have described an $r n c$ mutation in E. coli which appears to fulfil those criteria. In this mutant, designated D45A, an aspartic acid residue which is essential for the catalytic function of RNase III is changed to an alanine residue (Sun et al., 2004). D45A is essentially unable to cleave model substrates; the catalytic activity of the enzyme is $\sim 30000$-fold lower than that of the wild-type RNase III. Nevertheless, the D45A mutant is capable of binding an RNA substrate in a fashion that is comparable to that of the wild-type enzyme (Sun et al., 2004).
The aspartic acid residue identified by Sun et al. (2004) is conserved in bacterial RNases III, as shown in Fig. 1. Indeed there is strong conservation of several amino acids in the region of the proteins that contains the critical Asp residue. This observation suggested that mutating that Asp residue in $S$. coelicolor RNase III would produce a protein with properties similar to those of the D45A mutant in $E$. coli. As described in Methods, we constructed a mutant of S. coelicolor RNase III in which the Asp residue at position 70 was changed to Ala (D70A).

\section{The D70A protein lacks catalytic activity but retains the ability to bind an RNA substrate}

In addition to the construction of pJSE1855, we cloned the D70A gene into pET19B. This cloning attached a hexahistidine tag to the $\mathrm{N}$ terminus of the RNase III ORF. We overexpressed the protein and purified it by immobilized metal ion affinity chromatography. We then examined the activity of wild-type $S$. coelicolor RNase III and the D70A protein in RNA cleavage assays using an RNA substrate derived from the $S$. coelicolor $r p s O-p n p$ operon. This transcript, designated 5600 , is similar to the one used in our original characterization of the catalytic activity of S. coelicolor RNase III (Chang et al., 2005), and

S. coelicolor
W. succinogenes
A. aeolicus
B. subtilis
C. crescentus
C. diphtheriae
E. coli
G. sulfurreducens
H. pylori
M. tuberculosis
Synechocystis
T. maritima

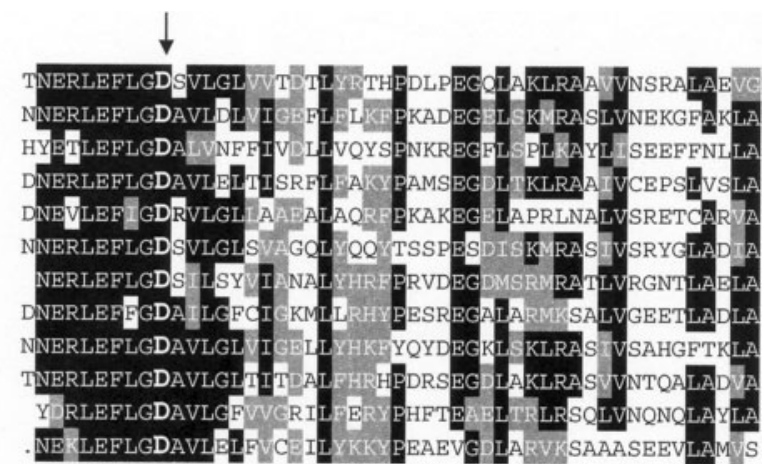

Fig. 1. PILEUP comparison (Devereux et al., 1984) of the region of bacterial RNases III containing the critical aspartic acid residue mutated in this study (arrow). Species shown are as follows: S. coelicolor, Wollinella succinogenes, Aquifex aeolicus, Bacillus subtilis, Caulobacter crescentus, Corynebacterium diphtheriae, E. coli, Geobacter sulfurreducens, Helicobacter pylori, Mycobacterium tuberculosis, Synechocystis, Thermotoga maritima. 
was also used by us in a kinetic analysis of PNPase (Chang et al., 2008). The left panel of Fig. 2 shows the results of cleavage assays using the 5600 transcript labelled internally with $\left[{ }^{32} \mathrm{P}\right] \mathrm{CTP}$ and wild-type RNase III. It can be seen that the enzyme cleaves this substrate effectively, as we have demonstrated previously (Chang et al., 2005). Cleavage produces two major bands, shown in Fig. 2 and representing cleavage on the $5^{\prime}$ and $3^{\prime}$ sides of an internal loop in the 5600 transcript (Chang et al., 2005). A third band, representing the apex of the stem-loop in the transcript, ran off the bottom of the gel shown in Fig. 2. In contrast, even at the highest concentration of $\mathrm{D} 70 \mathrm{~A}$ protein employed, no cleavage of this substrate was observed, even after $20 \mathrm{~min}$ of incubation at $37{ }^{\circ} \mathrm{C}$ (Fig. 2, middle panel). Amounts of enzyme up to $1 \mu \mathrm{g}$ did not lead to observable cleavage of the substrate (data not shown). Thus, as was the case for the D45A mutant in E. coli, our data indicate that the D70A change in S. coelicolor RNase III abolishes the catalytic activity of the enzyme.

To determine whether D70A retained the ability to bind RNAs, electrophoretic mobility shift assays were performed, as described in Methods. Results of such assays are shown in Fig. 3. The left panel of the figure shows that wild-type S. coelicolor RNase III binds the 5600 transcript effectively with 20-100 ng of enzyme in reaction mixtures. The right panel of the figure shows that D70A retains the ability to bind the substrate, at the same protein concentrations used for the wild-type enzyme. Although no attempt was made in these experiments to measure dissociation constants, the data of Fig. 3 suggest that D70A binds the 5600 transcript at least as well as does wild-type RNase III at comparable concentrations. Indeed, D70A appears in our hands to bind the substrate more effectively than the wild-type enzyme. Thus, our data indicate that like D45A, the D70A mutation abolishes the catalytic activity of $S$. coelicolor RNase III but not its ability to bind RNA substrates.

\section{The endoRNase activity of S. coelicolor is required for the regulation of act and red synthesis}

We tested the ability of JSE1880 and various derivatives thereof to produce two of the antibiotics normally elaborated by $S$. coelicolor, i.e. act and red. Fig. 4 shows the results of growth of the various strains on R2YE medium. It can be seen that M145 makes both act and red. The red antibiotic can be clearly seen after 3 days of incubation, and although both antibiotics are present after 5 days of incubation, the deep-blue colour of act prevents visualization of the red antibiotic at longer incubation times. As predicted, JSE1880 did not make detectable amounts of either antibiotic. The JSE1880 plate shown in Fig. 4 was incubated for 5 days. No act or red was detectable even after 7-10 days of incubation.

As shown on the top right in Fig. 4, a five-day incubation of pIJ8600/JSE1880 produced no detectable act. Interestingly, a small amount of red antibiotic was observed after 5 days of incubation, and the amount of antibiotic increased slightly in the presence of thiostrepton. We currently have no explanation for this observation. Restoring a wild-type copy of $r n c$ (pJSE1851) to JSE1880 also restored the ability of the resulting strain to produce both the act and red antibiotics. The tipA promoter was somewhat leaky in our hands, and it can be seen that significant amounts of red and some act were produced in a five-day incubation in the absence of thiostrepton. Both 5 and $15 \mu \mathrm{g}$ thiostrepton $\mathrm{ml}^{-1}$ stimulated production of act and red. The most straightforward interpretation of this observation is that thiostrepton induces transcription of the $r n c$ gene in pJSE1851, leading to the production of wild-type RNase III.

The bottom-right row of plates in Fig. 4 shows the results of the analysis of pJSE1855/JSE1880. Again, a small amount of red antibiotic was observed and the amount was highest

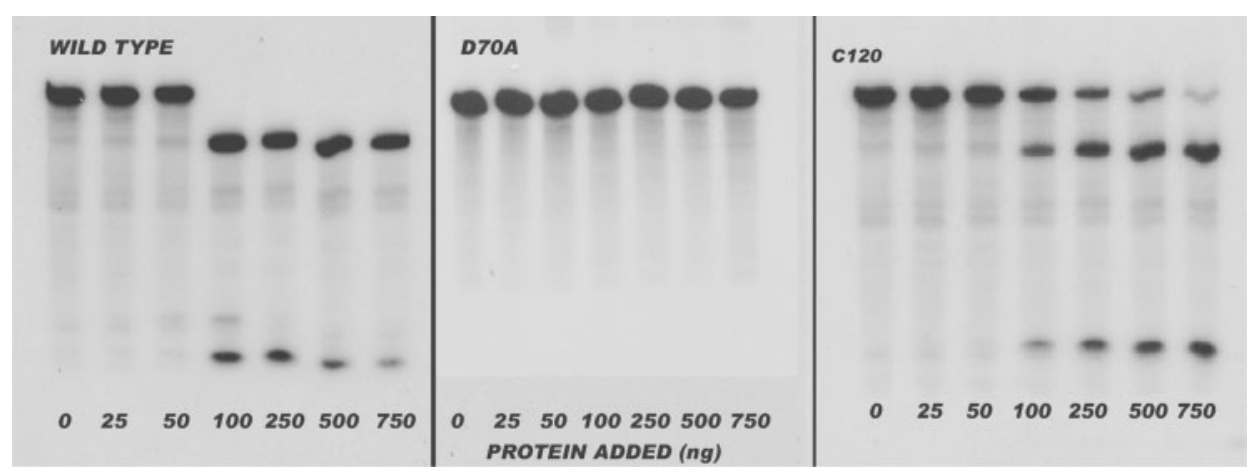

Fig. 2. RNA cleavage assays. Left panel, cleavage of the 5600 transcript with varying amounts of wild-type $S$. coelicolor RNase III; middle panel, cleavage of the transcript with the D70A mutant RNase III; right panel, cleavage of the transcript with the C120 mutant RNase III. Cleavage reactions were performed as described in Methods and the products were analysed on $5 \%$ ureapolyacrylamide gels. Bands were visualized by autoradiography of dried gels. 


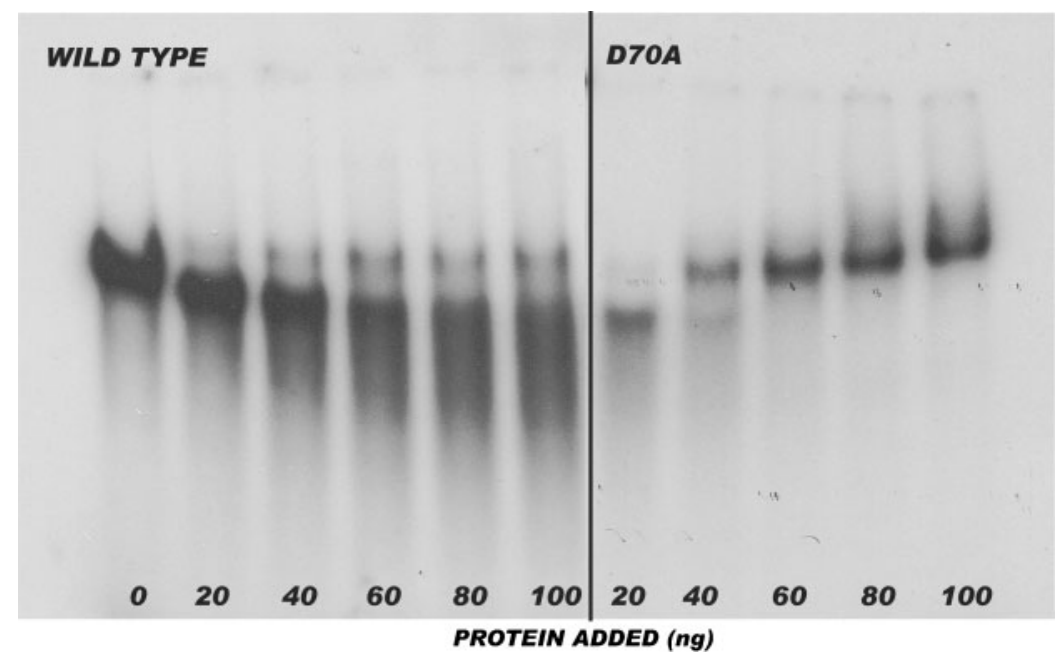

Fig. 3. Electrophoretic mobility shift assays. Left panel, assay of the binding of ${ }^{32} \mathrm{P}$-labelled 5600 transcript by wild-type $S$. coelicolor RNase III. Right panel, binding of the transcript by the D70A mutant RNase III. Binding assays and electrophoresis on $6 \%$ non-denaturing polyacrylamide gels were performed as described in Methods.

at $15 \mu \mathrm{g}$ thiostrepton $\mathrm{ml}^{-1}$. However, the amount observed for pJSE1855/JSE1880 was essentially the same as that observed in the control strain containing pIJ8600. No additional red was produced when plates were incubated for up to 7 days and no act was observed at any time (data not shown). Again, the most straightforward interpretation of this observation is that pJSE1855/JSE1880 does not produce the active RNase III which is required for the production of act and red. Results similar to these were observed when the relevant strains were grown on SFM medium.

The foregoing observations and their interpretation hinge on the assumption that both the wild-type $r n c$ and the D70A gene are expressed in the relevant constructs described above. To test this assumption, RNA was isolated from wild-type $S$. coelicolor mycelium and from mycelium of strains pIJ8600/JSE1880, pJSE1851/JSE1880 and

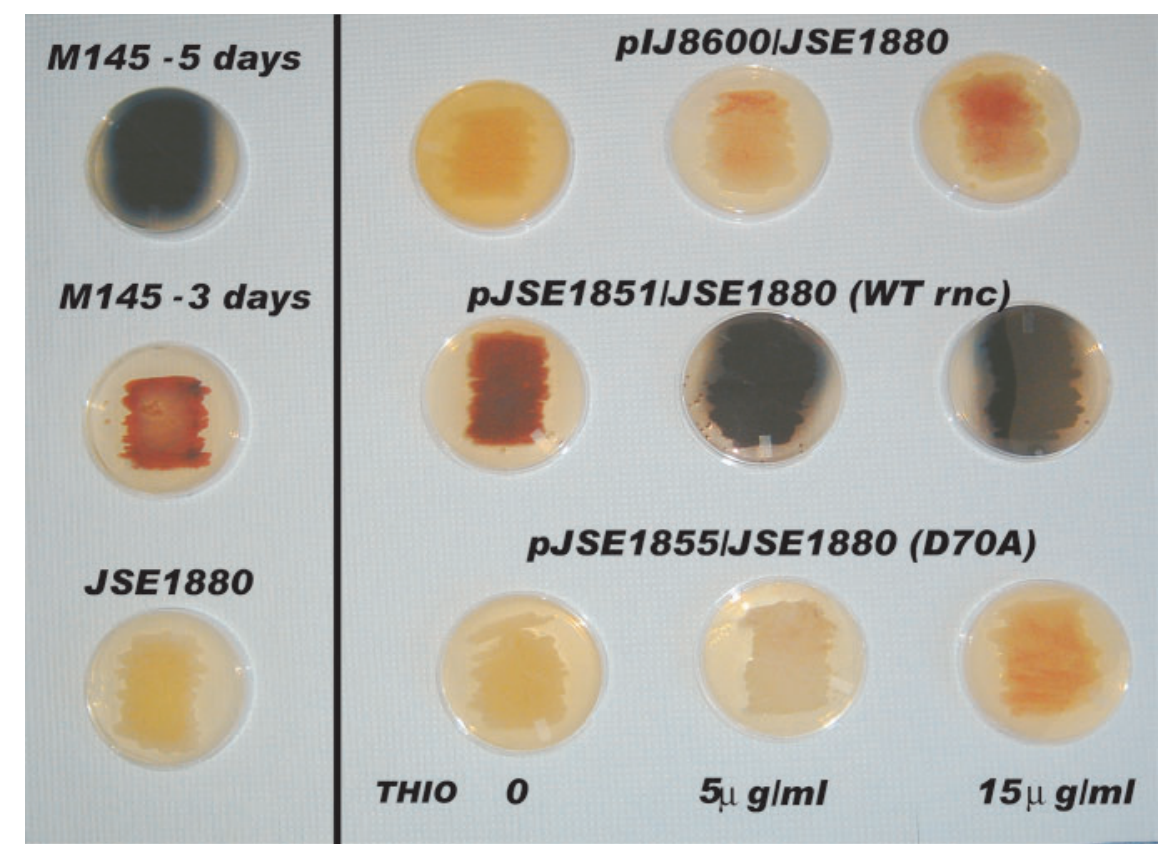

Fig. 4. Antibiotic production by wild-type and mutant strains of $S$. coelicolor. Spores were streaked to R2YE medium. Plates bearing the wild-type strain, M145, were incubated for 3 or 5 days to permit visualization of both the red and act antibiotics. All other plates were incubated for 5 days. Plates streaked with strains bearing derivatives of plJ8600 were incubated without thiostrepton or with 5 or $15 \mu \mathrm{g}$ thiostrepton $\mathrm{ml}^{-1}$ to induce expression from the tipA promoter. The JSE1880 plate contained viomycin $\left(30 \mu \mathrm{g} \mathrm{ml}^{-1}\right)$, while the plates bearing plJ8600 derivatives contained apramycin $\left(50 \mu \mathrm{g} \mathrm{ml}^{-1}\right)$. All plates contained nalidixic acid $\left(25 \mathrm{~g} \mathrm{ml}^{-1}\right)$ and nystatin $\left(315 \mathrm{U} \mathrm{ml}^{-1}\right)$. 
JSE1855/JSE1880, and the RNA was analysed by RT-PCR using $r n c$-specific primers as described in Methods. Fig. 5 shows the results of this analysis. Lane 2 shows the product obtained by PCR with M145 chromosomal DNA alone. Lane 3 shows that a product of the same size is obtained by RT-PCR using RNA isolated from M145. No such product is seen in lane 4, which shows the results of RT-PCR using RNA isolated from pJSE8600/JSE1880. The $r n c$-specific product is observed in lanes 5 and 6 , which show the results of RT-PCR using RNA isolated from pJSE1851/ JSE1880 and pJSE1855/JSE1880, respectively. Thus, the $r n c$ genes are expressed in those strains. Taken together, the plate assay and RT-PCR results lead to the conclusion that the catalytic activity of RNase III is required for its regulation of act and red production in S. coelicolor. In the absence of active RNase III, even when a form of the enzyme capable of RNA binding was present, no act or red was produced.

\section{The C120 mutant RNase retains enzymic activity}

The C120 mutant isolated in the pioneering studies of Champness and co-workers was not only used in their studies, it has also been used recently in a microarray study of the expression of genes involved in antibiotic synthesis (Huang et al., 2005b) and in an analysis of $r n c$ autoregulation in S. coelicolor (Xu et al., 2008). Given the extensive use of this mutant and the fact that Champness and coworkers observed a more drastic effect on antibiotic production in their rnc disruptant, as compared with C120 (Price et al., 1999), it was of interest to determine whether the $\mathrm{C} 120$ protein retained any enzymic activity in

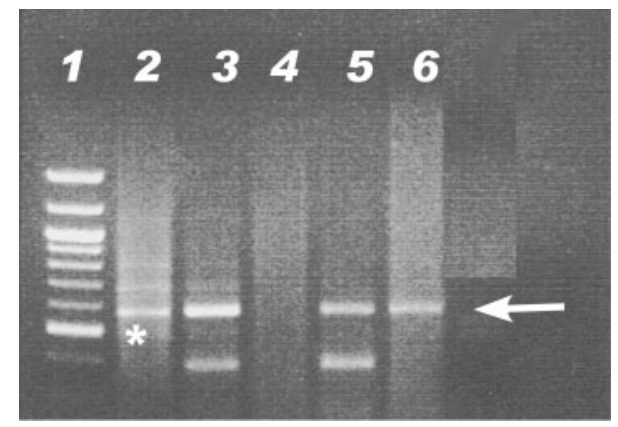

Fig. 5. RT-PCR analysis of RNAs isolated from $S$. coelicolor strains. RT-PCR was performed as described in Methods using random hexamers for reverse transcription and the MGabsBF1 and MGabsBR1 primers (Table 1) for PCR. Products were displayed on an agarose gel. Lane 1, size standards. The asterisk indicates the $1 \mathrm{~kb}$ standard band. Lane 2, PCR only with DNA from $S$. coelicolor M145; lane 3, RT-PCR with RNA from M145; lane 4, RT-PCR with RNA from pJSE8600/JSE1880; lane 5, RT-PCR with RNA from pJSE1851/JSE1880; lane 6, RT-PCR with RNA from pJSE1855/JSE1880. The arrow indicates the position of the band expected from PCR using the MGabsBF1 and MGabsBR1 primers. The lower band shown in lanes 3 and 5 is a PCR artefact. our assays. To this end, we reconstructed $\mathrm{C} 120$ as described in Methods and examined its activity using the 5600 transcript. Results of this analysis are shown in Fig. 2 (right panel). It can be seen that the $\mathrm{C} 120$ protein retains some enzymic activity, although it is not as active on a molar basis as our preparation of wild-type RNase III. We performed kinetic assays as described previously (Chang et al., 2005) to allow a more detailed comparison of the activities of the wild-type and mutant enzymes. The $k_{\text {cat }} / K_{\mathrm{m}}$ value for the wild-type enzyme was $2.76 \times 10^{6} \mathrm{M}^{-1} \mathrm{~min}^{-1}$, while the corresponding value for $\mathrm{C} 120$ was $3.26 \times 10^{4} \mathrm{M}^{-1}$ $\min ^{-1}$. Thus, the catalytic activity of the wild-type enzyme is 85 times greater than that of $\mathrm{C} 120$. The electrophoretic analysis suggests that the same products obtained from cleavage assays containing wild-type RNase III are produced by the C120 mutant enzyme (Fig. 2).

\section{DISCUSSION}

The autoregulation of $r n c$ expression in E. coli (Bardwell et al., 1989; Matsunaga et al., 1996, 1997) and Streptomyces (Xu et al., 2008) and the regulation of pnp expression by RNase III (Robert-Le Meur \& Portier, 1992, 1994) in E. coli are examples of the involvement of the catalytic activity of RNase III in the control of gene expression. In a study of the structure of the complex between Aquifex aeolicus RNase III and a model dsRNA substrate, Blaszczyk et al. (2004) proposed that RNase III can assume two different RNA binding modes, one which supports RNA processing and one which allows dsRNA binding but not RNA cleavage. They argue that the latter binding mode allows RNase III to regulate gene expression without dsRNA processing. There is evidence to support this hypothesis. Thus, Oppenheim et al. (1993) have shown that translation of the cIII mRNA in cells infected by bacteriophage $\lambda$ is strongly dependent on RNase III. They proposed that the cIII mRNA can adopt alternative structures, one of which $(\mathrm{ON})$ can be translated efficiently by $E$. coli ribosomes and one of which (OFF) has the Shine-Dalgarno sequence and the AUG initiation codon sequestered in a base-paired region and cannot be efficiently translated. They argued that RNase III can bind to the ON structure without cleaving it, thereby stabilizing that structure and facilitating the translation of the cIII mRNA. Dasgupta et al. (1998) isolated a mutant RNase III, designated Rnc70, which retained the ability to bind to dsRNAs but did not cleave them. They showed that multicopy expression of $r n c 70$ suppressed the cold-sensitive phenotype in an E. coli strain bearing the ssyA3 mutation. This result also indicates that RNase III can regulate gene expression, even when it cannot cleave RNA substrates, presumably via the ability of the enzyme to bind RNAs under these conditions.

The foregoing observations suggested to us the formal possibility that RNase III could regulate antibiotic production in $S$. coelicolor by binding to specific RNA targets without cleaving them. It was thus of critical importance, before exploring the mechanism of that 
regulation further, to determine whether the catalytic activity of the enzyme was necessary for antibiotic regulation. The most straightforward interpretation of the results presented here is that the catalytic activity of RNase III is required for its regulation of act and red synthesis in S. coelicolor. Replacing the wild-type protein with D70A, which can bind RNA substrates but cannot cleave them, was not sufficient to restore antibiotic production to JSE1880. We did not examine the effects of D70A on the production of the calcium-dependent antibiotic or on methylenomycin production.

We have also demonstrated that one of the mutations originally isolated by Champness and co-workers, C120, does not completely abolish the activity of RNase III. Although the mutation changes leucine to proline (Price et al., 1999), and would be expected to affect the tertiary structure of the protein in a significant fashion, we observed that the $\mathrm{C} 120$ protein was still able to cleave the 5600 transcript, albeit with an efficiency that was 85 -fold lower than that of the wild-type enzyme. The C120 enzyme appears to be more active in our assays, with the rspO-pnp transcript as substrate, than in the experiments described by $\mathrm{Xu}$ et al. (2008) using the absB transcript as substrate. Our findings, coupled with the observation of Champness and co-workers that antibiotic production is detectably reduced in the C120 mutant (Adamidis \& Champness, 1992), suggest that antibiotic synthesis in S. coelicolor is exquisitely sensitive to regulation by RNase III.

The question remains, what are the targets for RNase III regulation of antibiotic production in S. coelicolor? There are numerous studies which indicate that various antibiotic regulatory circuits are superimposed on each other in $S$. coelicolor (Bibb, 1996; Chater \& Hopwood, 1989; Huang et al., 2005a), so it is possible that RNase III is required for the processing of transcripts of regulatory genes that have already been identified. Champness and co-workers have shown, for example, that expression of the actII-orf4 and redD genes is dependent on RNase III (Aceti \& Champness, 1998), although there is currently no evidence that the transcripts of these genes are substrates for RNase III. It seems equally possible, however, that transcripts of other, as-yet-unidentified, genes are the actual targets for RNase III regulation of antibiotic production. Thus, RNase III might process transcripts for activators of antibiotic production. In the $a b s B$ mutant, those activators would not be produced, at least not in their active forms, and no antibiotics would be made. Alternatively, RNase III might be required to degrade the transcripts for repressors of antibiotic synthesis. In the abs $B$ mutant, those transcripts and their protein products would persist. A third possibility would involve effects of RNase III action on phosphate and nitrogen levels in S. coelicolor. Normally, RNase III cleavage coupled with the subsequent decay of target RNAs might affect the levels of those two nutrients. Abolishing RNase III activity might lead to decreases in phosphate and nitrogen levels, both of which are well known to affect the production of streptomycete anti- biotics (Bibb, 2005; Hillerich \& Westpheling, 2008; Rodriguez-Garcia et al., 2007). Experiments are in progress to examine these possibilities.

\section{ACKNOWLEDGEMENTS}

These studies were supported in part by a grant from the Emory University Research Committee.

\section{REFERENCES}

Aceti, D. J. \& Champness, W. (1998). Transcriptional regulation of Streptomyces coelicolor pathway-specific antibiotic regulators by the $a b s A$ and $a b s B$ loci. J Bacteriol 180, 3100-3106.

Adamidis, T. \& Champness, W. (1992). Genetic analysis of $a b s B$, a Streptomyces coelicolor locus involved in global antibiotic regulation. J Bacteriol 174, 4622-4628.

Bardwell, J. C., Regnier, P., Chen, S. M., Nakamura, Y., GrunbergManago, M. \& Court, D. L. (1989). Autoregulation of RNase III operon by mRNA processing. EMBO J 8, 3401-3407.

Bibb, M. J. (1996). 1995 Colworth Prize Lecture. The regulation of antibiotic production in Streptomyces coelicolor A3(2). Microbiology 142, 1335-1344.

Bibb, M. J. (2005). Regulation of secondary metabolism in streptomycetes. Curr Opin Microbiol 8, 208-215.

Blaszczyk, J., Gan, J., Tropea, J. E., Court, D. L., Waugh, D. S. \& Ji, X. (2004). Noncatalytic assembly of ribonuclease III with doublestranded RNA. Structure 12, 457-466.

Bralley, P. \& Jones, G. H. (2003). Overexpression of the polynucleotide phosphorylase gene ( $p n p)$ of Streptomyces antibioticus affects mRNA stability and poly(A) tail length but not ppGpp levels. Microbiology 149, 2173-2182.

Chang, S. A., Bralley, P. \& Jones, G. H. (2005). The $a b s B$ gene encodes a double-strand specific endoribonuclease that cleaves the readthrough transcript of the rspO-pnp operon in Streptomyces coelicolor. J Biol Chem 280, 33213-33219.

Chang, S. A., Cozad, M., Mackie, G. A. \& Jones, G. H. (2008). Kinetics of polynucleotide phosphorylase: comparison of enzymes from Streptomyces and Escherichia coli and effects of nucleoside diphosphates. J Bacteriol 190, 98-106.

Chater, K. F. \& Hopwood, D. A. (1989). Antibiotic biosynthesis in Streptomyces. In Genetics of Bacterial Diversity, pp. 129-150. Edited by D. A. Hopwood \& K. F. Chater. London: Academic Press.

Dasgupta, S., Fernandez, L., Kameyama, L., Inada, T., Nakamura, Y., Pappas, A. \& Court, D. L. (1998). Genetic uncoupling of the dsRNAbinding and RNA cleavage activities of the Escherichia coli endoribonuclease RNase III - the effect of dsRNA binding on gene expression. Mol Microbiol 28, 629-640.

Devereux, J., Haeberli, P. \& Smithies, O. (1984). A comprehensive set of sequence programs for the VAX. Nucleic Acids Res 12, 387-395.

Drider, D. \& Condon, C. (2004). The continuing story of endoribonuclease III. J Mol Microbiol Biotechnol 8, 195-200.

Flett, F., Mersinias, V. \& Smith, C. P. (1997). High efficiency intergeneric conjugal transfer of plasmid DNA from Escherichia coli to methyl DNA-restricting streptomycetes. FEMS Microbiol Lett 155, 223-229.

Gust, B., Challis, G. L., Fowler, K., Kieser, T. \& Chater, K. F. (2003). PCR-targeted Streptomyces gene replacement identifies a protein domain needed for biosynthesis of the sesquiterpene soil odor geosmin. Proc Natl Acad Sci U S A 100, 1541-1546. 
Hillerich, B. \& Westpheling, J. (2008). A new TetR family transcriptional regulator required for morphogenesis in Streptomyces coelicolor. J Bacteriol 190, 61-67.

Huang, J., Shi, J., Molle, V., Sohlberg, B., Weaver, D., Bibb, M J., Karoonuthaisiri, N., Lih, C J., Kao, C. M. \& other authors (2005a). Cross-regulation among disparate antibiotic biosynthetic pathways of Streptomyces coelicolor. Mol Microbiol 58, 1276-1287.

Huang, J., Shi, J., Molle, V., Sohlberg, B., Weaver, D., Bibb, M. J., Karoonuthaisiri, N., Lih, C. J., Kao, C. M. \& other authors (2005b). Cross-regulation among disparate antibiotic biosynthetic pathways of Streptomyces coelicolor. Mol Microbiol 58, 1276-1287.

Jarrige, A.-C., Mathy, N. \& Portier, C. (2001). PNPase autocontrols its expression by degrading a double-stranded structure in the $p n p$ mRNA leader. EMBO J 20, 6845-6855.

Ji, X. (2006). Structural basis for non-catalytic and catalytic activities of ribonuclease III. Acta Crystallogr D62, 933-940.

Kieser, Y., Bibb, M. J., Buttner, M. J., Chater, K. F. \& Hopwood, D. A. (2000). Practical Streptomyces Genetics. Norwich, UK: The John Innes Foundation.

Laemmli, U. K. (1970). Cleavage of structural proteins during the assembly of the head of bacteriophage T4. Nature 227, 680-685.

Matsunaga, J., Simons, E. L. \& Simons, R. W. (1996). RNase III autoregulation: structure and function of $r n c O$, the posttranscriptional “operator". RNA 2, 1228-1240.

Matsunaga, J., Simons, E. L. \& Simons, R. W. (1997). Escherichia coli RNase III $(r n c)$ autoregulation occurs independently of $r n c$ gene translation. Mol Microbiol 26, 1125-1135.

Nicholson, A. W. (1999). Function, mechanism and regulation of bacterial ribonucleases. FEMS Microbiol Rev 23, 371-390.

Oppenheim, A. B., Kornitzer, D., Altuvia, S. \& Court, D. L. (1993). Posttranscriptional control of the lysogenic pathway in bacteriophage lambda. Prog Nucleic Acid Res Mol Biol 46, 37-49.
Price, B., Adamis, T. \& Champness, W. (1999). A Streptomyces coelicolor antibiotic regulatory gene, absB, encodes an RNase III homolog. J Bacteriol 181, 6142-6151.

Robert-Le Meur, M. \& Portier, C. (1992). E. coli polynucleotide phosphorylase expression is autoregulated through an RNAase IIIdependent mechanism. EMBO J 11, 2633-2641.

Robert-Le Meur, M. \& Portier, C. (1994). Polynucleotide phosphorylase of Escherichia coli induces degradation of its RNase III processed messenger by preventing its translation. Nucleic Acids Res 22, 397403.

Rodriguez-Garcia, A., Barreiro, C., Santos-Beneit, F., Sola-Landa, A. \& Martin, J. F. (2007). Genome-wide transcriptomic and proteomic analysis of the primary response to phosphate limitation in Streptomyces coelicolor M145 and in a $\Delta$ phoP mutant. Proteomics 7, 2410-2429.

Sello, J. K. \& Buttner, M. J. (2008). The gene encoding ribonuclease III in Streptomyces coelicolor is transcribed during exponential phase and is required for antibiotic production and proper sporulation. J Bacteriol 190, 4079-4083.

Sun, J., Kelemen, G. H., Fernandez-Abalos, J. M. \& Bibb, M. J. (1999). Green fluorescent protein as a reporter for spatial and temporal gene expression in Streptomyces coelicolor A3(2). Microbiology 145, 22212227.

Sun, W., Li, G. \& Nicholson, A. W. (2004). Mutational analysis of the nuclease domain of Escherichia coli ribonuclease III. Identification of conserved acidic residues that are important for catalytic function in vitro. Biochemistry 43, 13054-13062.

Xu, W., Huang, J. \& Cohen, S. N. (2008). Autoregulation of AbsB (RNase III) expression in Streptomyces coelicolor by endoribonucleolytic cleavage of absB operon transcripts. J Bacteriol 190, 5526-5530.

Edited by: M. Paget 